\title{
Comparison between static and dynamic warm-up exercise regimes on lower limb muscle power
}

\author{
Jose Shelton ${ }^{1}$, G. V. Praveen Kumar ${ }^{2}$ \\ ${ }^{1}$ Victoria University, Melbourne, Australia; josenoel2008@gmail.com \\ ${ }^{2}$ School of Biotechnology, Chemical \& Biomedical Engineering, VIT University, Vellore, India; gidi99_5611@yahoo.co.in
}

Received 26 April 2009; revised 17 May 2009; accepted 10 June 2009.

\begin{abstract}
Aim: The purpose of this study was to compare static and dynamic warm-up regimes on lower limb muscle power and thereby the performance of the individual. Methodology: Twenty eight (28) subjects were assigned into groups consisting of 2 members. From each group, 1 subject performed the static stretching and the other subject performed dynamic stretching as warm-up. This was followed by non-counter movement jumps on a force platform and the vertical jump heights were recorded. Data were analysed using one-way ANOVA and paired t-test at $\mathbf{0 . 0 5}$ alpha. Result: The results showed that dynamic stretching as warm-up causes significant increase $(p=0.01)$ in the vertical jump height as compared to static stretching $(p=0.03)$. Discussion: The increase in vertical jump height could be related to the increase in force production which plays an important role during the vertical jump test. On the other hand the decrease in vertical jump height following static stretching could be attributed to a decrease in the force production in the muscles. Conclusion: Dynamic warm-up increases the vertical lump height, whereas static stretching decreases the jump height of the athlete.
\end{abstract}

Keywords: Static Stretching; Dynamic Stretching; Force Production; Post Activation Potentiation

\section{INTRODUCTION}

The primary aim of exercise physiologists, personal trainers, bio-mechanical engineers and sports scientists is to monitor and increase the performance levels of the athletes under their training. When it comes to training and prescribing exercises, there is always a debate between the types of stretching that are being used as warm up before activity. This could also be used to check the performance of the athlete owing to the particular type of stretching.

Static stretching involves holding the muscle in the stretched position for some time. This type of stretching has been used as a traditional method of warm up as well as performance enhancement for quite some time now. But research performed by Rosenbaum and Hennig (1995) [1], shows that static stretching decreases peak force by $5 \%$ and rate of force production by $8 \%$, there by actually decreasing muscle strength. Static stretching of calf, hamstrings and quadriceps reduces the peak vertical velocity of a vertical jump according to studies done by Knudson et al., 2000 [2]. Studies done by Kokkonen et al., 1998 [3], have documented a rather harmful effect of acute static stretching, that it actually decreases the performance of those tasks where success is related to maximal force development. Further studies by McNeal and Sands, 2003 [4], with younger populations have also illustrated impairment in jumping performance in teenagers following static stretching.

Dynamic stretching consists of functional based exercises which use sport specific movements to prepare the body for movement. It consists of controlled leg and arm swings that are taken gently to the limits of range of motion. Studies done by Fredrick G. A., 2000 [5] have shown the effectiveness of dynamic stretching, as this increases core temperature, muscle temperature, elongates the muscles and stimulates the nervous system, thereby decreasing the chances of injury. Faigenbaum et al., 2005 [6] studied dynamic warm-up versus static stretching in different age groups and a variety of athletes. And found that compared to static stretching, dynamic warm up increases flexibility and also improved performances among children for vertical jump. Longjump performance also improved in the dynamic warmup. Studies by Duncan M. J. and Woodfield L. A., 2006 [7] suggest that there may be some advantage to performing a low to moderate dynamic warm up protocol prior to activities that require high power outputs.

The purpose of this study is to find out which type of stretching exercise used as warm-up affects lower limb muscle power and therefore affects performance of an individual. 


\section{METHODOLOGY}

\subsection{Subjects}

Twenty eight moderately trained subjects (16 male and 12 female) ranging in the age group of 20 to 35 years were taken for the study. They were randomly divided into groups consisting of 2 members. From each group, one subject performed the static stretching and the other subject performed the dynamic stretching as part of the warm up.

\subsection{Procedure of Data Collection: Baseline Measurement}

Both the groups performed an initial non-counter movement jump with both hands on the hips on a force platform and the vertical jump height was recorded.

The subjects in both the groups were made to jog 12 laps (up to $60 \% \mathrm{VO}_{2} \max$ ) up and down in the corridor after which the heart rate (Carotid artery) was recorded. Then they performed the first non-counter movement jump on the force platform and the vertical jump heights were recorded.

\subsection{Stretching Protocol}

The static stretching group subjects actively performed some static calf, hamstrings, quads, gluteal and hip flexor stretching exercises for 2 repetitions 30 seconds each, for both the legs.

While the dynamic stretching group subjects performed some dynamic stretching exercises like tip-toe walking, forward and backward leg swings, sagittal plane leg swings, walking knee pull ups, walking lunges with hip rotation and walking quads stretches for $2 \times 10$ repetitions for both legs.

\subsection{Post Stretch Measurement}

Then the second heart rate (Carotid artery) was recorded for both the groups. After which they performed the second non-counter movement jump on the force platform and the vertical jump heights were recorded. Then the subjects were asked to remain standing, without doing any activity for 10 minutes. Then they performed the third and final non-counter movement jump on the force platform and the vertical jump heights were recorded. Finally the vertical jump heights and heart rate readings for both the static and dynamic groups were recorded.

\subsection{Data Analysis}

Descriptive statistics of range, mean and standard deviation were computed on all data. One way ANOVA was calculated across the recording of both the groups. A paired t-test was computed to compare the static and dynamic stretching groups. Level of significance was set at 0.05 alpha.

\section{RESULTS}

The mean and standard deviation of the jump heights of the subjects in both the static and dynamic stretching groups are shown in Tables $\mathbf{1}$ and $\mathbf{2}$.

The static stretching group showed a decrease of $0.61 \%$ in the final jump as compared to the dynamic

Table 1. Jump heights of the subjects in the static stretching group $(\mathrm{N}=14)$

\begin{tabular}{ccccccc}
\hline Subjects & $\begin{array}{c}\text { Initial } \\
\text { Jump }\end{array}$ & $\begin{array}{c}\text { Jump } \\
\text { I }\end{array}$ & $\begin{array}{c}\text { Jump } \\
\text { II }\end{array}$ & $\begin{array}{c}\text { Jump } \\
\text { III }\end{array}$ & HR I & $\begin{array}{c}\text { HR } \\
\text { II }\end{array}$ \\
\hline $\mathrm{n}=1$ & 0.139 & 0.184 & 0.138 & 0.175 & 104 & 96 \\
$\mathrm{n}=2$ & 0.174 & 0.164 & 0.186 & 0.202 & 128 & 100 \\
$\mathrm{n}=3$ & 0.134 & 0.108 & 0.123 & 0.152 & 180 & 128 \\
$\mathrm{n}=4$ & 0.086 & 0.145 & 0.127 & 0.137 & 172 & 126 \\
$\mathrm{n}=5$ & 0.132 & 0.132 & 0.107 & 0.141 & 168 & 88 \\
$\mathrm{n}=6$ & 0.288 & 0.331 & 0.309 & 0.293 & 152 & 100 \\
$\mathrm{n}=7$ & 0.265 & 0.208 & 0.149 & 0.183 & 138 & 102 \\
$\mathrm{n}=8$ & 0.255 & 0.309 & 0.28 & 0.291 & 144 & 88 \\
$\mathrm{n}=9$ & 0.124 & 0.223 & 0.205 & 0.21 & 150 & 100 \\
$\mathrm{n}=10$ & 0.176 & 0.201 & 0.193 & 0.158 & 92 & 64 \\
$\mathrm{n}=11$ & 0.15 & 0.216 & 0.18 & 0.158 & 140 & 94 \\
$\mathrm{n}=12$ & 0.084 & 0.083 & 0.055 & 0.047 & 132 & 96 \\
$\mathrm{n}=13$ & 0.345 & 0.345 & 0.203 & 0.233 & 144 & 92 \\
$\mathrm{n}=14$ & 0.149 & 0.171 & 0.192 & 0.206 & 150 & 112 \\
$\mathrm{AVG}$ & 0.172 & 0.201 & 0.174 & 0.184 & 142.42 & 99 \\
STDEV & 0.077 & 0.079 & 0.066 & 0.063 & 24.04 & 15.95 \\
\hline
\end{tabular}

Table 2. Jump heights of the subjects in the dynamic stretching group $(\mathrm{N}=14)$.

\begin{tabular}{ccccccc}
\hline Subjects & $\begin{array}{c}\text { Initial } \\
\text { Jump }\end{array}$ & $\begin{array}{c}\text { Jump } \\
\text { I }\end{array}$ & $\begin{array}{c}\text { Jump } \\
\text { II }\end{array}$ & $\begin{array}{c}\text { Jump } \\
\text { III }\end{array}$ & HR I & $\begin{array}{c}\text { HR } \\
\text { I }\end{array}$ \\
\hline $\mathrm{n}=1$ & 0.35 & 0.27 & 0.406 & 0.338 & 120 & 104 \\
$\mathrm{n}=2$ & 0.119 & 0.156 & 0.149 & 0.163 & 156 & 141 \\
$\mathrm{n}=3$ & 0.251 & 0.146 & 0.244 & 0.121 & 176 & 148 \\
$\mathrm{n}=4$ & 0.135 & 0.129 & 0.156 & 0.187 & 132 & 128 \\
$\mathrm{n}=5$ & 0.224 & 0.159 & 0.15 & 0.19 & 144 & 120 \\
$\mathrm{n}=6$ & 0.12 & 0.01 & 0.118 & 0.12 & 168 & 120 \\
$\mathrm{n}=7$ & 0.188 & 0.201 & 0.214 & 0.212 & 152 & 140 \\
$\mathrm{n}=8$ & 0.098 & 0.092 & 0.107 & 0.102 & 160 & 132 \\
$\mathrm{n}=9$ & 0.099 & 0.092 & 0.105 & 0.101 & 160 & 130 \\
$\mathrm{n}=10$ & 0.237 & 0.272 & 0.304 & 0.264 & 164 & 128 \\
$\mathrm{n}=11$ & 0.164 & 0.179 & 0.156 & 0.168 & 180 & 108 \\
$\mathrm{n}=12$ & 0.282 & 0.282 & 0.259 & 0.266 & 118 & 100 \\
$\mathrm{n}=13$ & 0.187 & 0.169 & 0.189 & 2 & 100 & 88 \\
$\mathrm{n}=14$ & 0.245 & 0.231 & 0.353 & 0.296 & 140 & 128 \\
$\mathrm{AVG}$ & 0.192 & 0.170 & 0.207 & 0.323 & 147.85 & 122.5 \\
STDEV & 0.075 & 0.077 & 0.094 & 0.488 & 23.46 & 17.09 \\
\hline
\end{tabular}




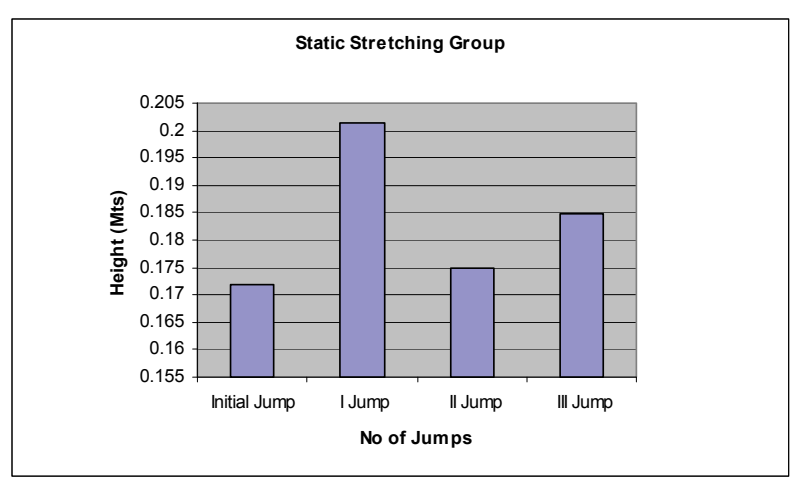

Figure 1. Graph showing the differences in vertical jump height in the static stretching group.

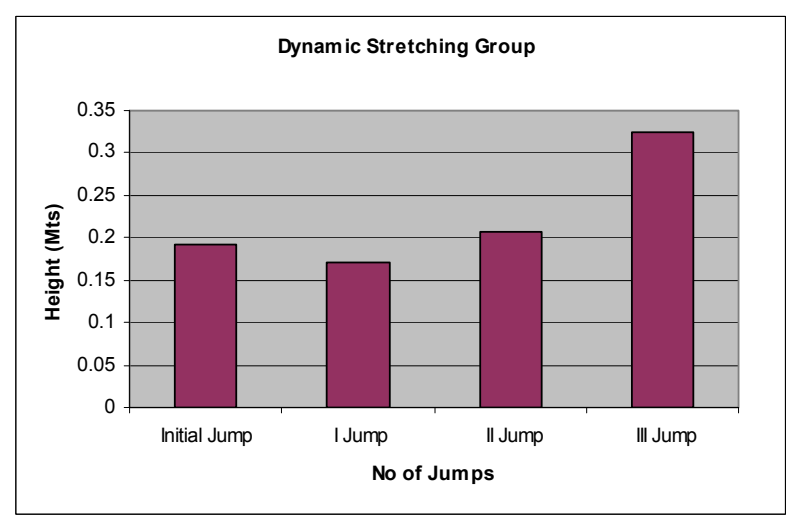

Figure 2. Graph showing the differences in vertical jump height in the dynamic stretching group.

stretching group which showed an increase of $13.06 \%$, as shown in Figures 1 and 2.

\section{DISCUSSION}

As the result shows dynamic warm up can definitely increase the vertical jump height and therefore significantly influences fitness performance, as compared to the group that did static stretching as warm up. These findings are similar to the studies done by Duncan and Woodfield 2006 [7] and Faigenbaum et al., 2005 [6] which show that dynamic stretching increases flexibility as well as muscle power.

Among the subjects who did static stretching, from Figure 3, we can see that there is a decrease in jump height between the first and second jumps. This shows that static stretching might actually reduce force production, which is similar to the studies done by Rosenbaum and Hennig, 1995 [1]. The main muscles involved in a vertical jump are the calf, quadriceps and hamstrings. These muscles were part of the static stretching protocol of the warm up. On the other hand, we can see an increase in the vertical jump height between second and third jumps, this change could be because of the ten minutes rest period in between the jumps. And this rest period would have given time for the muscles to recover after the period of static stretching. This implies that static stretching actually causes a decrease in the force production in these muscles as also shown in the studies by Kokkonen et al., 1998 [3] and Knudson et al., 2000 [2]. Therefore the performance of the activity (vertical jump height) is also decreased as a result of static stretching which is also similar to the studies done by McNeal J. and Sands W., 2003 [4]. The reason for this decrease in performance could be attributed to an increase in the musculo-tendinous unit (MTU) compliance, leading to a decrease in the MTU ability to store elastic energy in its eccentric phase as reported by Fletcher IM, Jones B, 2004 [8]. The above evidences suggest that static stretching prior to activity is not the best solution. Static stretching does not necessarily lead to a decrease in injury but may actually decrease the force production and thereby decrease the vertical jump height for the athlete.

On the other hand we can see from Figure 4, there is a significant increase in the vertical jump height in the group that did dynamic stretching as part of the warm up, which is similar to studies done by Faigenbaum et al, 2005 [6]. Studies by Duncan and Woodfield, 2006 [7] have suggested that performing pre-event dynamic warm up protocols may create an optimal environment for ex-

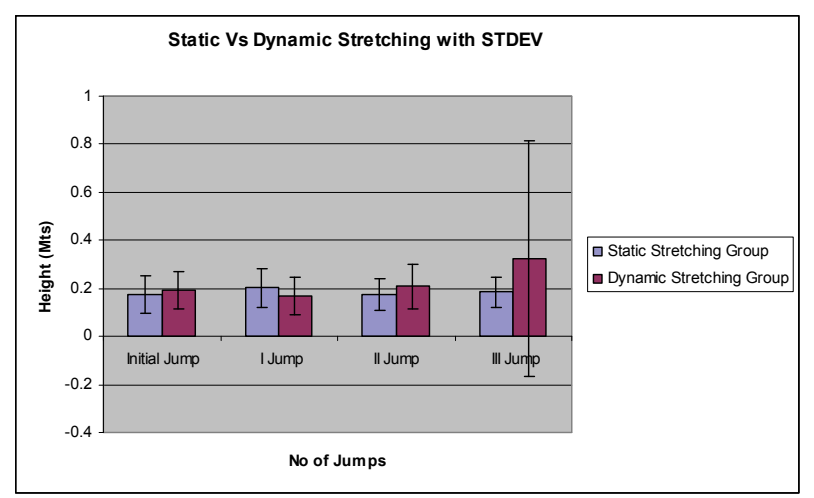

Figure 3. Graph showing the differences in vertical jump height between the static and dynamic stretching groups (Using AVERAGE +/- STDEV).

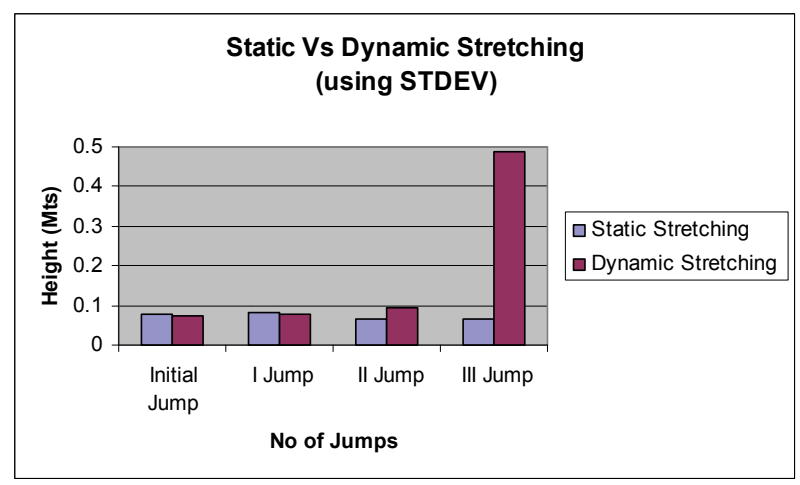

Figure 4. Graph showing the differences in vertical jump height between the static and dynamic stretching groups (Using STDEV values). 
plosive force production by enhancing neuromuscular function. This occurrence has been termed the 'postactivation potentiation' (PAP) [9] and is believed to increase the rate of force development, thereby increasing speed and power production. This finding was similar to the study done by Faigenbaum et al., 2005 [6]. Dynamic warm up activities used in the study may have influenced the excitability of fast twitch motor units and therefore readied these units to play a more significant role during the vertical jump test. However no tests of neuromuscular activation were performed in this study. Neuromuscular activation studies can be done in future to measure the excitability of fast twitch motor units. The results of the current study suggest that there may be some advantage to performing a low to moderate dynamic warm up protocol prior to activities that require high power outputs. And the increase in vertical jump height following dynamic warm up compared to static warm up is considerable.

Faigenbaum et al. 2005 [6] in his study also says that the evidence supporting the injury-reducing and performance-enhancing potential of static stretching is presently lacking. So it may be desirable to perform dynamic stretching during the warm up period and static stretching during the cool down. The purpose of warm up exercise is to warm-up the body, but static stretching seems to cause cool down of the body.

\section{CONCLUSIONS}

From the above study it can be concluded that the effect of dynamic stretching as warm up has the following benefits. Dynamic Stretching increase force production prior to activity, which in turn can improve the vertical jump height of the athlete.

As exercise physiologists and sports scientists our main objective is to decrease the injury levels and increase the performance levels of the athletes. And the above evidences from related literature suggest that dy- namic stretching is the best type of stretching that can be performed during warm-up in order to increase the jump height of the athlete and to increase performance levels of the athlete. From the findings of the study in order to increase the vertical jump height of the athlete we can recommend a sports performance program that includes dynamic activities during warm up and static stretching as part of the cool down.

\section{REFERENCES}

[1] Rosenbaum, D. and E. M. Hennig (1995) The influence of stretching and warm-up exercises on Achilles tendon reflex activity. Journal of Sport Sciences, 13(6), 481-90.

[2] Knudson, D., Bennet, K., Corn, R., Leick, D., Smith, C., (2000) Acute effects of stretching are not evident in the kinematics of the vertical jump. Research Quarterly for Exercise and Sport, 71(1-Supplement), A-30.

[3] Kokkonen, J., Nelson, A. G., Cornwell, A. (1998) Acute muscle stretching inhibits maximal strength performance. Research Quarterly for Exercise and Sport, 69, 411-415.

[4] McNeal, J. and Sands, W., (2003) Acute static stretching reduces lower extremity power in trained children. Pediatric Exercise Science, 15, 139-145.

[5] Frederick, G.A. (2001) Baseball part-1 dynamic flexibility. Strength \& Conditioning Journal, 23(1), 21-30.

[6] Faigenbaum, A.D., Bellucci, M.A., Bernieri, A, Bakker, B., Hoorens, K., (2005) Acute effects of different warm up protocols on fitness performance in children. Journal of Strength \& Conditioning Research, 19(2), 376-381.

[7] Duncan, M.J. and Woodfield, L.A., (2006) Acute effects of warm up protocol on flexibility and vertical jump in children. Journal of Exercise Physiologyonline, online, 9 (3), 9-16.

[8] Fletcher, I.M. and Jones, B., (2004) The effects of different warm up stretch protocols on 20metre sprint performance in trained rugby union players. Journal of Strength and Conditioning Research, 18(4), 885.

[9] Sale, D., (2002) Postactivation potentiation: Role in human performance. Exercise Sport Science Review, 30(3), 138-143. 